\title{
COMPARISON OF TWO DENTURE ADHESIVES ON MANDIBULAR COMPLETE DENTURE RETENTION AND PATIENT SATISFACTION
}

\author{
Amr A. Rady *
}

\begin{abstract}
Introduction: One of the main problems faced by conventional complete denture wearers is the retention and stability of their dentures during function. To overcome this problem, many attempts have been tried including invasive surgical techniques, implants, suction systems and the least offensive denture adhesives. Dentists have prescribed a range of adhesives of highly varied composition and efficacy.
\end{abstract}

Purpose: The present clinical study evaluates the effectiveness of two adhesives in improving complete denture retention as well as patients' satisfaction.

Material and Methods: An in vivo clinical study is made of 20 patients with complete mandibular dentures to evaluate the retention provided by two commercial complete denture adhesives (Poligrip and Fittydent). A digital force meter scale was used to measure retention strength (in grams). Patients were asked to answer a simple questionnaire before and after using the tested adhesives regarding their acceptance to the retention and chewing ability.

Results: The results obtained indicate that retention is enhanced using both adhesives, no significant difference was detected between the two adhesives and patients approved the enhancement of their denture retention and chewing ability in presence of both adhesives.

Conclusion: within the limitation of this study it can be concluded that denture adhesives efficiently improve the denture retention.

\section{INTRODUCTION}

Despite the dropping rate of complete edentulism due to significant success of implant-supported prostheses, there are still several edentulous patients who still utilize complete dentures considering it esthetically acceptable and certainly of low $\operatorname{cost}^{(l)}$. Successful complete dentures integrate with the functions of the masticatory system and the psychological acceptance of the patient which is greatly relying on the quality of retention which in turn depends on complex interaction between adhesion, cohesion, atmospheric pressure, surface tension, and viscosity ${ }^{(2)}$.

\footnotetext{
* Lecturer of Removable Prosthodontics, Faculty of Oral and Dental Medicine, Cairo University.
} 
Although, there are some problematic situations where providing optimum retention to the denture may be a problem; prosthodontists tend to neglect the advantages of using denture adhesives thinking that the use of adhesives is an indication of poor denture retention, which in turn implies improper denture fabrication. Consequently, denture adhesive use by a patient is considered a negative annotation on the dentist's clinical skill. In contrast to this undesirable attitude toward the use of adhesives, evidence is now stressing that these products can be beneficial as part of denture care ${ }^{(3)}$.

With proper information and instruction, denture adhesives can be a useful tool in increasing retention and stability, enhanced comfort, improved function, and in providing psychological satisfaction of edentulous patients. They should not be used unconsciously without proper guidance and instructions from the dentists to recompense for denture faults even though adhesives enhance denture performance ${ }^{(4)}$.

In certain circumstances, the use of denture adhesives becomes vital such as demanding patient, gross maxillofacial defects, dry mouth, poor ridge anatomy or relations and in public like solicitors, actors, and politicians. Moreover, alveolar bone resorption causes dentures to gradually become loose; thus, relining or new denture construction are the only remedy. Denture adhesives fill gaps caused by shrinking bone and give temporary relief from the loosening dentures ${ }^{(5)}$. Added to that, the use of denture adhesives becomes compulsory for the improvement of function in other situations such as severely atrophic edentulous ridges, abused or hypertrophied tissue covering the denture foundation, changes in saliva quality or quantity due to medications or age and patients having lack of neuromuscular control as in stroke and Parkinsonism ${ }^{(6)}$.

Denture adhesives have proved useful during different stages of denture fabrication such as during recording of jaw relation with denture bases, during the try-in of teeth as well as during insertion of immediate dentures ${ }^{(7)}$.

It has been demonstrated that adhesives improve patient's satisfaction, bite force, masticatory efficacy, retention, and stability of dentures ${ }^{(6,8)}$, moreover, these advantages have been correlated to patients suffering from xerostomia ${ }^{(9)}$. Added to that, adhesives reduce three-dimensional and rotational denture movements and chewing times in old and new dentures therefore improve chewing function ${ }^{(10)}$. However, this benefit may deceive patients into ignoring the need for seeking professional help even if the dentures become ill-fitting so dentists should emphasize on periodic denture check up to minimize the use of denture adhesives.

It was always believed that adhesive material swells 50 to 150 percent by volume in the presence of water, filling in spaces between the prosthesis and the tissues. On the contrary, modern adhesives provide strong bio adhesive and cohesive forces via carboxyl groups that form electrovalent bonds providing stickiness and viscosity of the medium lying between the denture and the basal seat and eliminating the voids between the denture base and the tissues ${ }^{(11)}$.

\section{AIM OF THE STUDY}

The study evaluates the effectiveness of two commercially different denture adhesives in improving mandibular complete denture retention and patient's satisfaction.

\section{Materials and Method}

The study was carried out on 20 patients wearing removable mandibular complete dentures with no previous experience of using denture adhesives, independently of how long the dentures had been used. After the obtainment of informed consent in all cases, retention was determined without adhesive at first to be used as control. 
Patients were asked to sit in an upright position with the head resting firmly on the headrest and instructed to maintain maximum, non-forced intercuspation for 5 minutes. After this time, and with the mouth open and the lower lip relaxed to avoid losing peripheral seal, the tip of a digital force meter (Eagle OF PRC, ELT 3000) (Fig.1) was covered with plastic sleeve and placed at the labial margin of the dentures, in the recess of the lower buccal frenum. Traction was then applied perpendicular to the occlusal plane until the dentures were dislodged, the maximum retention force being registered by the force meter (Fig. 2).

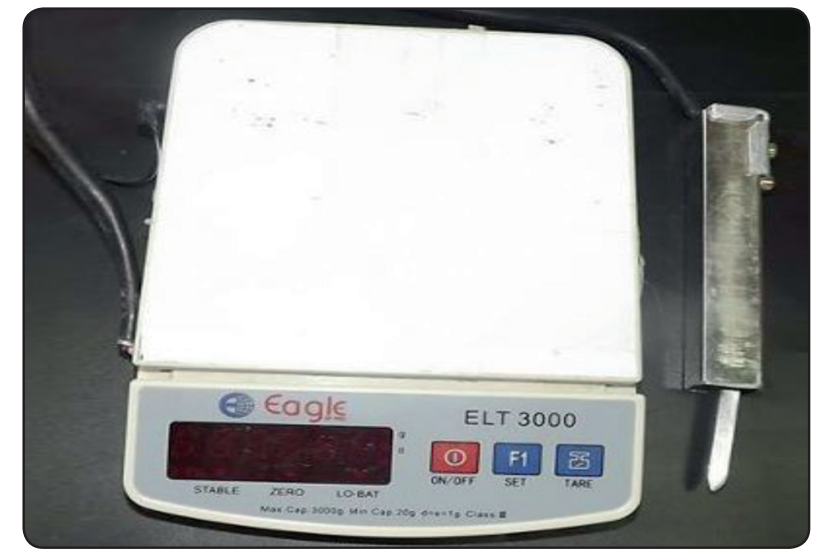

Fig. (1) Digital force meter used in the study

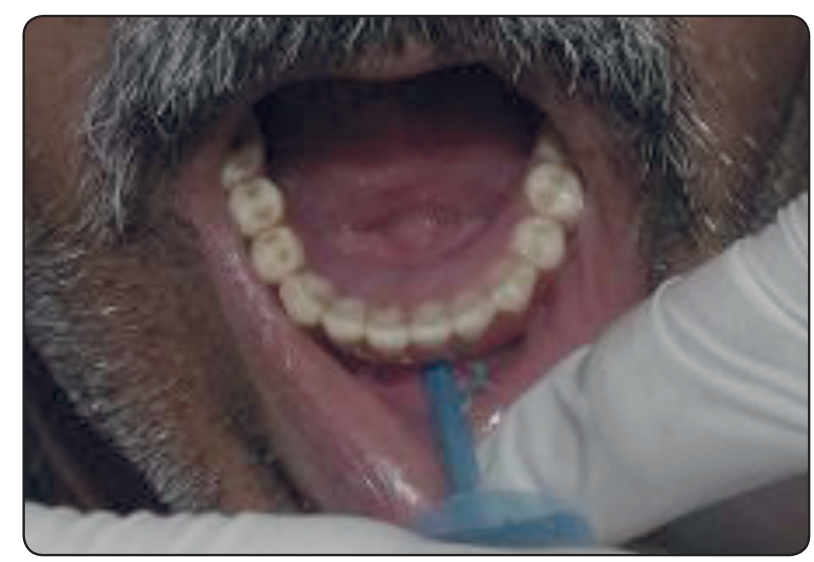

Fig. (2) Tip of the force meter placed at the labial frenum before traction

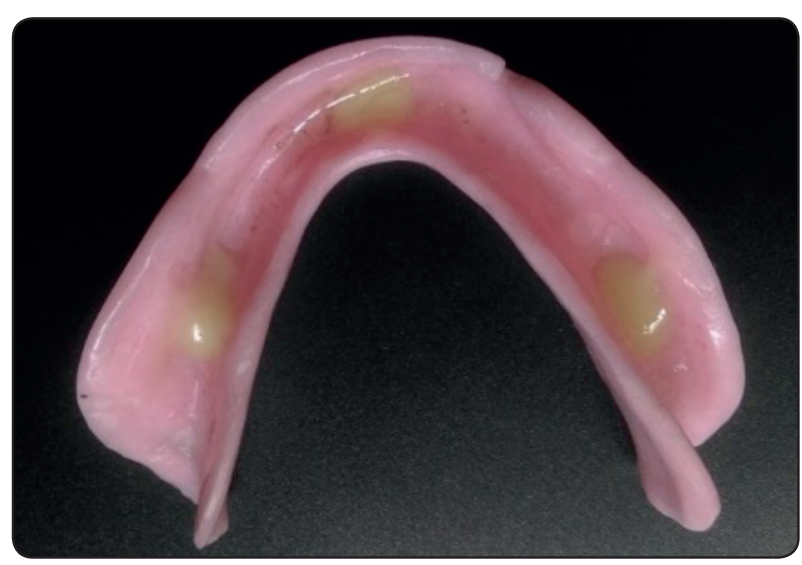

Fig. (3) Adhesive distribution in the denture fitting surface

Two complete denture adhesives were selected, supplied in the form of creams and with a similar formulation: one imported type Poligrip (GLAXOMITHKLINE, RTP NC, Ireland) and one locally available type Fittydent (Fittydent Int. GMBH, Pinkafeld, Austria).

After recording retention of the mandibular dentures, dentures were cleaned and dried before applying the first adhesive in short strips away from the denture borders, especially to the depth of the tissue-bearing surface. The same amount of adhesive was used in all tests, distributing the material in three pea-sized equivalent increments in the anterior and posterior zones, in accordance with the instructions of the manufacturer (Fig.3). Patients were instructed to close the jaws together several times (in centric occlusion) to evenly distribute the adhesive below the denture in a thin layer and remove any additional excess that expresses into the cheek or tongue spaces before applying the tip of the force meter to measure the force needed to displace the dentures as described above.

This procedure was repeated three times for each product, without adding further amounts of adhesive, and waiting one minute in occlusion after each measurement. After completing the study with each adhesive, patients were asked to swish their mouth 
and remove any adhesive residue from the denture and mouth with warm water and a soft brush before applying the other type of adhesive to eliminate any possible summing of effects among materials. All measurements were made by the same investigator. Thus, each patient was subjected to three measurements of retention strength without adhesive (control values), together with three measurements involving each of the adhesives. The averages of the three radings were calculated and tabulated for statistical analysis.

\section{Denture Satisfaction}

To evaluate satisfaction about the use of adhesives, patients were asked to answer a simple questionnaire which was developed for this study, where participants graded their acceptance for retention and chewing with their dentures before the use of the adhesive using the scale from 1 to 5 (from 1 = dissatisfaction to $5=$ excellent). one of the tested adhesives was given to the patients to use for a period of one week and then attend the clinic to answer a similar questionnaire. After that, the participants were given the second type of adhesive to use for a similar period before returning to provide their response on the questionnaire. All instructions concerning the correct application and cleaning of the denture adhesives were given to the

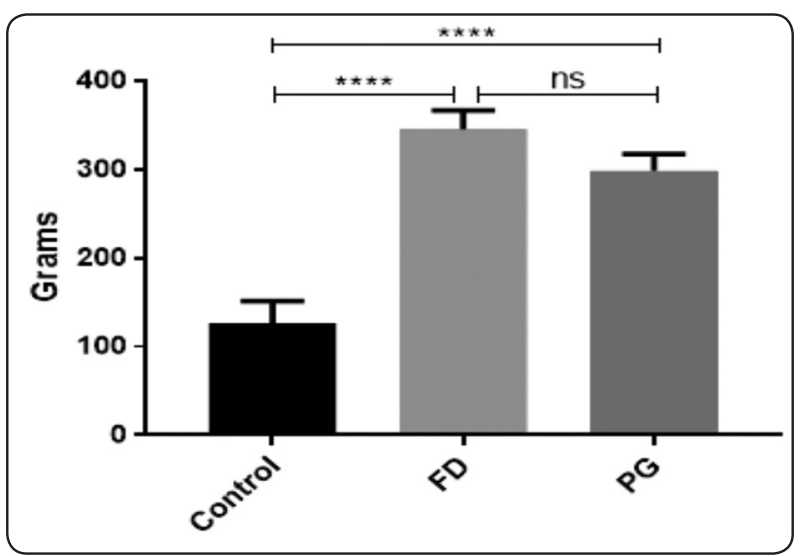

patients based on the modification done to Adisman $\operatorname{advices}^{(7,12)}$

Results were shown as mean \pm SEM (standard error of means). Statistical significance for the parametric data was investigated using one-way ANOVA with Tukey (comparisons between all groups) pairwise tests. In non-parametric data Kruskal-Wallis followed by Dunn's multiple comparison were the tests of choice. The calculations were performed with the statistical software package GraphPad Prism (version 7). P values $\leq 0.05$ were considered statistically significant.

\section{RESULTS}

Three recordings were obtained from each patient who was included in the study (control, Fittydent adhesive and Poligrip adhesive). ANOVA showed an overall significance between the tested groups. Additionally, Tukey multiple comparison test which revealed a non-significant difference between the two adhesives groups $(\mathrm{p}=0.2785)$, recorded an extremely significant increase in the retention force between each individual adhesive group and the control group $(\mathrm{p}<0.0001)$.

To assess the patient's satisfaction to the tested adhesives in terms of retention and chewing, a simple questionnaire was designed and given to

\begin{tabular}{|l|c|l|l|}
\hline \multicolumn{2}{|l|}{ ANOVA summary } & \\
\hline $\mathrm{F}$ & 28.39 \\
\hline P value & $<0.0001$ \\
\hline P value summary & $* * * *$ \\
\hline Tukey's m.c.t & $\begin{array}{c}\text { Mean } \\
\text { Diff. }\end{array}$ & Summary & $\begin{array}{c}\text { Adjusted P } \\
\text { Value }\end{array}$ \\
\hline Control vs. FD & -219 & $* * * *$ & $<0.0001$ \\
\hline Control vs. PG & -171.6 & $* * * *$ & $<0.0001$ \\
\hline FD vs. PG & 47.4 & $\mathrm{~ns}$ & 0.2785 \\
\hline
\end{tabular}

Fig. (4) Bar chart and tables showing extremely significant difference in retention of complete dentures when fitty dent (FD) and poligrip (PG) adhesives were used. The two adhesives did not show significant difference when compared to each other. $\mathrm{N}=15$ patients ( 3 readings/each patient). 
these patients before and after use of the adhesives. Scaling the patient's feedback on retention (from 1 = dissatisfaction to $5=$ excellent) was verified with Kruskal-Wallis test that showed an overall significance between the tested groups. Additionally, Dunn`s multiple comparison test revealed a non-significant difference between the two adhesives groups $(\mathrm{p}=0.5654)$ and recorded an extremely significant satisfaction from using the Fittydent $(\mathrm{p}<0.0001)$ compared to a significant satisfaction when the Poligrip was used $(\mathrm{p}=0.0146)$, all compared to the control group where patients did not use any adhesives.

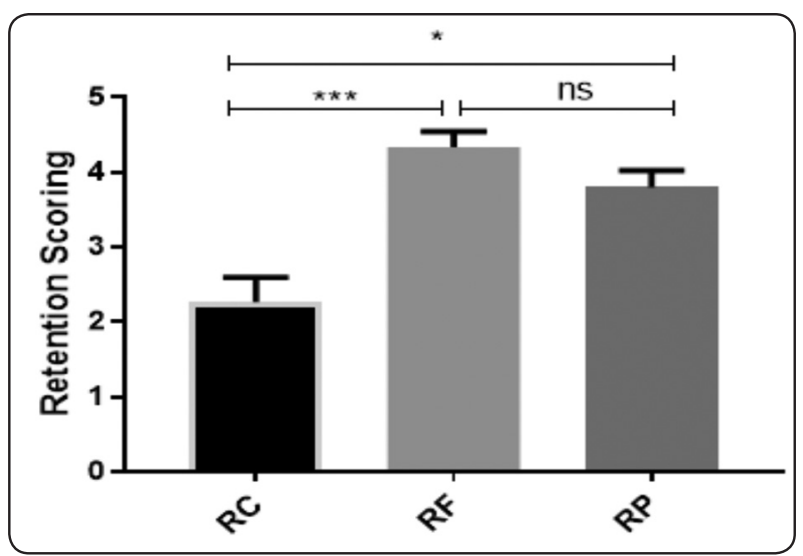

Scaling the patient's feedback on chewing with their dentures was verified with Kruskal-Wallis test that showed an overall significance between the tested groups. Additionally, Dunn's multiple comparison test revealed a non-significant difference between the two adhesives groups $(\mathrm{p}=0.3448)$ and recorded an extremely significant satisfaction from using the Fittydent $(\mathrm{p}<0.0001)$ compared to a very significant satisfaction when the Poligrip was used( $\mathrm{p}=0.0085)$, all compared to the control group where patients did not use any adhesives.

\begin{tabular}{|l|l|}
\hline Kruskal-Wallis test & \\
\hline P value & 0.0001 \\
\hline P value summary & $* * *$ \\
\hline
\end{tabular}

\begin{tabular}{|l|l|l|}
\hline Dunn's m. c. t & Summary & Adjusted P Value \\
\hline RC vs. RF & $* * *$ & 0.0001 \\
\hline RC vs. RP & $*$ & 0.0146 \\
\hline RF vs. RP & ns & 0.5654 \\
\hline
\end{tabular}

Fig. (5) Bar chart and tables showing no significant difference between patient's satisfaction with complete denture retention when Fittydent (FD) and Poligrip (PG) adhesives were used. Extremely significant satisfaction from using the Fittydent compared to a significant satisfaction when the Poligrip was used, all compared to the control group where patients did not use any adhesives. $\mathrm{N}=15$ patients.

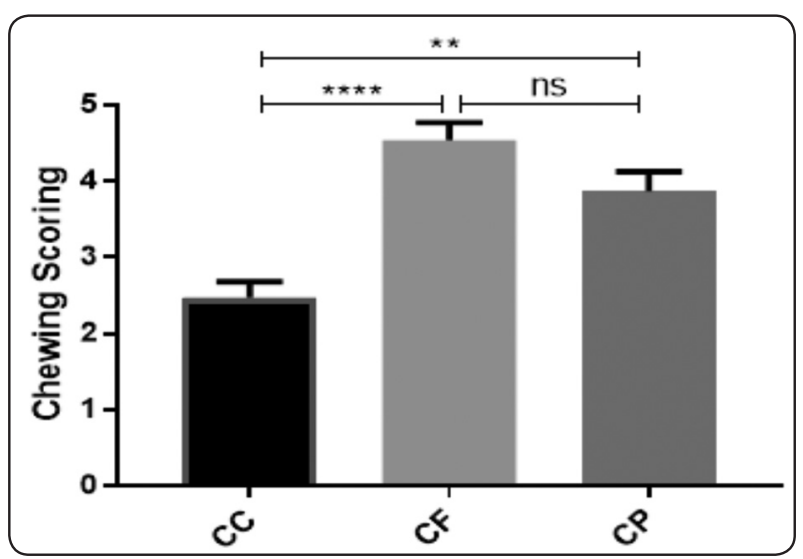

\begin{tabular}{|l|l|}
\hline Kruskal-Wallis test & \\
\hline P value & $<0.0001$ \\
\hline P value summary & $* * * *$ \\
\hline
\end{tabular}

\begin{tabular}{|c|c|c|}
\hline $\begin{array}{c}\text { Dunn's multiple } \\
\text { comparisons test }\end{array}$ & Summary & $\begin{array}{c}\text { Adjusted P } \\
\text { Value }\end{array}$ \\
\hline CC vs. CF & $* * * *$ & $<0.0001$ \\
\hline CC vs. CP & $* *$ & 0.0085 \\
\hline CF vs. CP & ns & 0.3448 \\
\hline
\end{tabular}

Fig. (6) Bar chart and tables showing no significant difference between patient's satisfaction with complete denture chewing when Fittydent (FD) and Poligrip (PG) adhesives were used. Extremely significant satisfaction from using the Fittydent compared to a very significant satisfaction when the Poligrip was used, all compared to the control group where patients did not use any adhesives. $\mathrm{N}=15$ patients. 


\section{DISCUSSION}

The study was carried on the mandibular dentures because most of the edentulous patients suffer with its retention problems rather than the maxillary ones. The patients were included independently of how long the dentures had been worn or of their quality ${ }^{(13)}$.

An ideal denture adhesive is described as nontoxic, nonirritating, and biocompatible with the oral mucosa and not promoting microbial growth. Furthermore, it should be odorless, tasteless, and easy to apply and to remove from the tissuebearing surface of dentures Various forms of adhesives are available in the market however, cream was preferred to powder because the former has a reduced tendency to be washed away in oral fluids and its effect remains for a longer time post insertion $^{(14)}$.

Successful complete denture therapy depends on excellent fabrication of the prosthesis and effective management of patient, the most skillful practitioners may find it difficult to satisfy the patient's expectations for stability and retention of the denture and it is often considered appropriate to prescribe a denture adhesive for these patients. Denture adhesives act as an aid to edentulous patients, and various research studies suggest that its use significantly decreased displacement of mandibular and maxillary dentures during chewing, biting and speaking. In coincidence with literature, the results of this study based on both clinical examination and patient's opinions, indicate that the tested adhesives increase effectively the retention of complete dentures ${ }^{(7,13,15,16)}$.

One of the main goal of prosthetic treatment is to restore masticatory function in conventional denture wearers who suffer from decreased oral proprioception, alterations in chewing dynamics that occur with prosthetic devices and reduced denture stability and retention which is further compounded by physiologic bone resorption of the denturebearing ridge ${ }^{(17)}$. Again, the responses of the patients towards the tested adhesives were favouring their use as it improved their ability to chew and this is in accordance with multiple studies and surveys ${ }^{(9,10,17)}$. This may be attributed to the fact that improved retention and stability results in an increase in the force that can be applied during chewing, leading to fewer strokes.

\section{CONCLUSION}

Within the limitation of this study it can be concluded that denture adhesives efficiently improve the denture retention as well as patient's satisfaction. However, more in vivo investigations are necessary in dental literature.

\section{REFERENCES}

1. G.E. Carlsson, R. Omar, The future of complete dentures in oral rehabilitation. A critical review. J Oral Rehabil 37 , 143-156 (2010).

2. D. O. Marin et al., Effect of a denture adhesive on the satisfaction and kinesiographic parameters of complete denture wearers: a cross-over randomized clinical trial. Braz Dent J 25, 391-398 (2014).

3. S. L. Yankell, Overview of research and literature on denture adhesives. Compend Contin Educ Dent Suppl 4, S18-21 (1984).

4. P. R. Kumar et al., Denture Adhesives in Prosthodontics: An Overview. J Int Oral Health 7, 93-95 (2015).

5. J. E. Grasso, J. Rendell, T. Gay, Effect of denture adhesive on the retention and stability of maxillary dentures. J Prosthet Dent 72, 399-405 (1994).

6. I. Duqum, K. A. Powers, L. Cooper, D. Felton, Denture adhesive use in complete dentures: clinical recommendations and review of the literature. Gen Dent 60, 467-477; quiz p. 478-469 (2012).

7. J. Psillakis, Denture adhesives usage in removable prosthodontics. Dent Today 22, 90-93 (2003).

8. S. Papadiochou, I. Emmanouil, I. Papadiochos, Denture adhesives: a systematic review. J Prosthet Dent 113, 391-397.e392 (2015). 
9. Z.A. Bogucki, P. Napadlek, T. Dabrowa, A Clinical Evaluation Denture Adhesives Used by Patients With Xerostomia. Medicine 94, e545 (2015).

10. S. Hasegawa, T. Sekita, I. Hayakawa, Effect of denture adhesive on stability of complete dentures and the masticatory function. J Med Dent Sci 50, 239-247 (2003).

11. D. Felton et al., Evidence-based guidelines for the care and maintenance of complete dentures: a publication of the American College of Prosthodontists. J Am Dent Assoc 142 Suppl 1, 1s-20s (2011).

12. I. K. Adisman, The use of denture adhesives as an aid to denture treatment. J Prosthet Dent 62, 711-715 (1989).

13. J. F. Manes, E. J. Selva, A. De-Barutell, K. Bouazza, Comparison of the retention strengths of three complete denture adhesives: an in vivo study. Med Oral Patol Oral Cir Bucal 16, e132-136 (2011).

14. F. Ghani, D. C. Picton, Some clinical investigations on retention forces of maxillary complete dentures with the use of denture fixatives. J Oral Rehabil 21, 631-640 (1994).

15. C. A. Munoz et al., A clinical study to evaluate denture adhesive use in well-fitting dentures. J Prosthodont 21, 123 129 (2012).

16. D. Quiney, W. Nishio Ayre, P. Milward, The effectiveness of adhesives on the retention of mandibular free end saddle partial dentures: An in vitro study. J Dent 62, 64-71 (2017).

17. T. M. Goncalves, F. C. Viu, L. M. Goncalves, R. C. Garcia, Denture adhesives improve mastication in denture wearers. Int J Prosthodont 27, 140-146 (2014). 